\title{
RESPONSABILIDAD SOCIAL CON VALOR COMPARTIDO CONSTITUYEN VENTAJAS COMPETITIVAS EN EMPRESAS PERUANAS Y MEXICANAS
}

\author{
SOCIAL RESPONSIBILITY WITH SHARED VALUE CONSTITUTES COMPETITIVE \\ ADVANTAGES IN PERUVIAN AND MEXICAN COMPANIES \\ Ana María Gutiérrez HubY* \\ Docente Principal de la Facultad de Ciencias Contables \\ Universidad Nacional Mayor de San Marcos - UNMSM / Lima - Perú \\ Ma Eugenia Amador Murguia** \\ Docente de la Universidad de Guadalajara - CULAGOS / México \\ [Recepción: Setiembre de 2015/ Conformidad: Octubre 2015]
}

\section{RESUMEN}

En este artículo se tiene por objetivo profundizar y poner al alcance de los involucrados en el ámbito empresarial de Perú y México, el conocimiento de la responsabilidad social con valor compartido. El propósito es sensibilizar en torno al desarrollo de una gestión empresarial responsable. Entre las conclusiones más significativas, cabe mencionar: la incipiente implementación de prácticas responsables pese a la proliferación de información sobre la temática; el ejercicio del liderazgo en las prácticas responsables con valor compartido es practicado mayoritariamente en las grandes empresas; la existencia de interés y predisposición hacia la aplicación de prácticas responsables por parte de la PYMES; un crecimiento en el interés en esta corriente por parte de las universidades y centros de formación empresarial y finalmente, la falta de apoyo de los gobiernos que aplican una regulación que impone una normatividad tributaria poco favorable al crecimiento empresarial. Es necesaria la consolidación del mercado de la responsabilidad social empresarial con valor compartido en ambos países, así como la capacidad de la sociedad para reaccionar ante las acciones positivas o negativas de las empresas.

\section{Palabras clave:}

Responsabilidad social empresarial; valor compartido; ventajas competitivas; gestión empresarial.

\begin{abstract}
This article aims to deepen and provide awareness of the social responsibility with shared value to those involved in the business area of Peru and Mexico. We propose to raise awareness about the development of responsible corporate management. Among the most significant findings we include: the incipient implementation of responsible practices despite the proliferation of information on the subject, the exercise of leadership in responsible practices with shared value is applied mainly in large companies, the existence of interest and predisposition towards implementing responsible practices by the SME, a growth in the interest in this trend by universities and corporate training centers, and finally the lack of support from governments implementing a regulation that imposes tax regulations unfavorable to business growth. A market consolidation of corporate social responsibility shared value is necessary in both countries; as well as the ability of society to respond to positive or negative actions of enterprises.
\end{abstract}

\section{Keywords:}

Corporate social responsibility; shared value; competitive advantages; business management.

\footnotetext{
* Doctora en Administración. Magister en Gestión Empresarial - UNMSM. Email: anamaria_01053@yahoo.es.

** Docente de la Universidad de Guadalajara - Centro Universitario de Los Lagos. México.

*** Colaboradora: Elsa Violeta Rafael Díaz.
} 


\section{INTRODUCCIÓN}

La razón de ser de la empresa ha sido y es la de servir. Habiendo sido creada por personas con el propósito de satisfacer las necesidades de la sociedad, esta ha sido su característica de siempre, servir al bien común a través de la producción de bienes y servicios; no obstante, en un determinado momento atribuido al sistema económico capitalista, las empresas se apartaron de este principio haciendo primar en su gestión el aspecto económico: la inversión y el logro de mayores utilidades por sobre otros aspectos como el bienestar de sus trabajadores, el medio ambiente, la comunidad, ignorando los efectos que su accionar y su proceso productivo generaban en la sociedad.

La falta de una cultura del consumo por parte de la sociedad, constituyó un importante aspecto que contribuyó en la agudización de esta situación ya que los criterios para las decisiones de compra, primordialmente contemplaban: el precio y la accesibilidad a la adquisición del producto; de esta manera, si un producto reunía las condiciones de ser barato, de fácil acceso y de calidad relativamente aceptable, era suficiente para decidir su compra pasando por alto la exigencia de calidad y la forma en que hubiese sido producido, considerándose por ello que la empresa se comportaba como una entidad desconectada del acontecer y bienestar del entorno en el que se desarrollaba, razón por la cual el rol de la empresa en la sociedad empezó a ser cuestionada fuertemente.

En este sentido, se incorporaron nuevos elementos en el análisis de los beneficios de la empresa y en la decisión de compra de los consumidores, entre ellos la profundización en la preocupación por el medio ambiente, los desastres de la naturaleza ocasionados por los procesos productivos, las formas de transportar productos, entre otros, son aspectos que han tenido efectos significativos en la conciencia de la colectividad mundial; así mismo, los temas de agenda mundial puestas en discusión en congresos y conferencias importantes como la de Río 92 en la que se desarrolló el tema del calentamiento global y la necesidad de contar con políticas públicas y privadas relacionadas al manejo ambiental y por otra parte, los efectos sociales provocados por las empresas en las comunidades en las que operaban, ampliaron el es- pectro de elementos a tomar en cuenta en el análisis, conformándose movimientos de vigilancia ambiental y agrupaciones de consumidores responsables que empezaron a demandar un nuevo tipo de comportamiento por parte de las empresas, agudizando la sensibilidad general hacia el tema de la responsabilidad empresarial con la sociedad.

Esta demanda de cambio se favoreció con la intensificación de la globalización, la explosión de los medios informativos, las redes sociales, canales que propalaron la percepción de la cada vez mayor disminución de los recursos naturales, que alertaron el no consumir en demasía y mucho menos, considerando los niveles de pobreza prevalecientes en muchos países en desarrollo.

El cambio demandado dio lugar a otra manera de gestionar por parte de las empresas, forjándose a partir de ello conceptos como: bien común, proyección social, compromiso con el entorno, responsabilidad social. Con la adopción de la responsabilidad social como nuevo modelo de gestión, los aspectos que se modifican en la nueva concepción de empresa lo constituyen la valoración de su dimensión cultural, la dimensión humana de la organización y la incorporación del concepto de cooperación en su dinámica interna y externa, configurándose tres tipos de capitales intervinientes en los procesos de la empresa: el físico, el humano y el social.

En este sentido, la adopción de la responsabilidad social empresarial ha constituido un factor de mejoramiento del entorno para los negocios, por el aumento del aprecio por la calidad de vida, por el reconocimiento del poder económico de las empresas, por la maduración de una sociedad exigente y participativa y por los cambios en la concepción y administración de las empresas, en consecuencia, al empresariado le ha tocado entender, cada vez con mayor certeza, que no es posible hacer buenos negocios si no se tiene presente el factor de responsabilidad social.

En la actualidad esta responsabilidad social ha evolucionado al incorporar la concepción del valor compartido, cuya definición viene a abarcar no solo lo que las empresas hacen con sus beneficios, sino también la forma en la que ellos hacen. Esto va más allá de la filantropía, el cumplimiento y la orientación 
de como las empresas gestionan sus derechos económicos, sociales, e impactos ambientales, así como sus relaciones en todos los ámbitos clave en donde tiene influencia: el lugar de trabajo, el mercado, la cadena de suministro, la comunidad y el ámbito de la política pública.

Por ello, puede afirmarse que la responsabilidad social ha adquirido una enorme relevancia, en especial en América Latina, en donde se presenta imprescindible por la necesidad sobre todo del sector privado de contribuir al desarrollo sostenible y más equitativo en la lucha contra la desigualdad y a favor de la inclusión económica, entendiéndose que toda empresa requiere operar en un entorno que le sea favorable y toda sociedad requiere de fuentes que le generen oportunidades de desarrollo.

Considerando el protagonismo que ha cobrado esta temática en la actualidad a nivel mundial, resulta necesario plantear algunas interrogantes en el contexto de la realidad de los países de esta región; en especial, Perú y México: ¿en qué medida se presenta esta interrelación entre empresa y sociedad?, ¿qué motivaría a las empresas interesarse en internalizar y asumir una política de responsabilidad social empresarial y más aún de una responsabilidad social con valor compartido?; en este marco de comportamiento responsable empresarial, ijuegan algún papel los principios y los valores éticos, la cultura de la calidad, la innovación? ¿resulta costoso para las empresas el asumir esta manera de gestionar?, ¿cuándo es el mejor momento en que una empresa deba plantearse el asumir esta manera de gestionar?. Siendo beneficiosa para una empresa esta nueva manera de gestionar, tal como se enuncia en la literatura revisada ipodría asumirse entonces que al menos el 50\% de las empresas de Perú y México seguirían esta línea de gestión?, de no ser así ¿cuáles serían las razones por la que aún no se evidenciaría en la realidad?, ¿qué se ha hecho o qué se viene haciendo en la actualidad en materia de responsabilidad social y valor compartido?, ¿cuáles son las ventajas competitivas que se obtendrían de poner en práctica esta tendencia?, ¿los miembros de estas organizaciones tienen interiorizada la responsabilidad social de manera que ello le permita a la organización desarrollar valor compartido con la sociedad?, ¿es factible la intervención del Estado en la generación de una conducta de responsabilidad social con valor compartido por parte de las empresas?

Estas interrogantes planteadas surgen al abordar el presente tema cuyo propósito final es sensibilizar al empresariado en general y a todos los actores involucrados en el medio, tanto de Perú como de México, incluyéndose a sus docentes universitarios y estudiantado pertenecientes a las carreras del área empresarial. En este enfoque de responsabilidad social con valor compartido se busca contribuir a una gestión empresarial responsable en estos países, poniendo a su alcance conocimientos y experiencias que pueden ayudar a generar riqueza, empleo y a la vez, bienestar social y medioambiental con la intención de sumar esfuerzos para mejorar la situación económica y social, en ambos ámbitos.

\section{ASPECTOS DIFERENCIALES ENTRE LA RESPONSABILIDAD SOCIAL CLÁSICA Y LA RESPONSABILIDAD SOCIAL CON VALOR COMPARTIDO}

De la literatura revisada en torno a Responsabilidad Social Empresarial (RSE) y el Valor Compartido (VC) se repara en las siguientes teorías: 1) Teoría de la Actuación Social por parte de la Empresa, 2)Teoría de la Creación de Valor para el Accionista, 3) Teoría de los Grupos de Interés y la 4) Teoría de la Ciudadanía Corporativa.

Según la primera teoría mencionada "Actuación Social por parte de la Empresa", la empresa se encarga de generar en la comunidad donde se desenvuelve, bienes no solamente económicos; sino también, busca minimizar los impactos negativos que pudiera generar maximizando sus resultados positivos; la segunda teoría: "Creación de Valor para el Accionista", esta teoría es cortoplacista y no considera ninguna responsabilidad social más allá de la obligación de solo maximizar valor para el accionariado.

Por su parte la tercera teoría: "Grupos de Interés”, definiéndose como grupos de interés a cualquier grupo o individuo que puedan afectar o ser afectados por las actividades de las empresas, según Freeman (1984), Donaldson y Preston (1995), esta teoría señala que las empresas no pueden ignorar el contexto donde se desarrollan puesto que están 
interconectadas a una red de relaciones con un gran número de individuos e instituciones denominadas grupos de interés o stakeholders, y por último, la cuarta teoría: "Ciudadanía Corporativa”, identifica más colectivos con los que la empresa se relaciona, aparte de los accionistas y admite cierta legitimidad a las expectativas que esos colectivos pueden tener en relación a la empresa, constituyendo estas en una suerte de contrato tácito entre ambos, empresa y diferentes colectivos.

Según lo señalan Porter y Kramer, el valor compartido pone los problemas ambientales y de la comunidad en el centro mismo de la gestión de las empresas sugiriendo gastar recursos para hacer las cosas bien, así mismo, establece la forma de tener un mejor desempeño económico al impactar positivamente en la sociedad.

Es por eso, que el valor compartido es un concepto que parte de una evidencia indiscutible: en el mundo que surge en estas décadas, las utilidades de las empresas deben ir a la par del progreso social y el desarrollo sostenible de la comunidad, que debe ser promovida por dichas empresas, y los resultados de las investigaciones lideradas por el propio Porter son concluyentes: "las estrategias de valor compartido son más sostenibles como ventajas competitivas que las mejoras al producto o la racionalización de costos".

De acuerdo a sus autores Porter y Kramer, la estrategia del valor compartido, implica el abandono de la mentalidad cortoplacista de exigir utilidades inmediatas, entendiendo que se presentarán oportunidades si se piensa ampliamente en el beneficio social. Se trata entonces de asumir conciencia de que hay que reformular la forma en la que aseguramos la rentabilidad y entendemos la gestión. En este sentido, las empresas que no se adapten a las necesidades de las sociedades que impactan, tarde o temprano sucumbirán.

\section{FORMAS PARA LA GENERACIÓN DE VALOR COMPARTIDO CON GRUPOS DE INTERÉS}

Según lo sostienen Porter y Kramer, existen tres estrategias clave para que una organización sea capaz de generar valor compartido con sus grupos de interés: Una primera forma es "reconcebir productos y mercados". Es decir, innovar con el producto o servicio que brinda la empresa, de manera que satisfaga una necesidad social ya que siempre existirá una oportunidad para la apertura de nuevos mercados que atiendan los requerimientos de clientes tradicionalmente ignorados.

Ello quiere decir que, la identificación y atención de las necesidades no consideradas por los mercados, es en donde está la clave para optar por la redefinición de los productos o servicios de la empresa, propiciando con ello la creación de valor compartido.

Una segunda manera, es redefinir la productividad en la cadena de valor, esta se refiere a buscar oportunidades de éxito a través del ejercicio de prácticas innovadoras empresariales que tengan en cuenta los impactos sociales, ambientales y económicos en el entorno, lo cual involucra, hallar nuevos caminos para desarrollar estas prácticas, como procurar compras a pequeñas empresas o negocios locales o utilizar nuevos modelos de distribución para los productos, ahorrando en el uso de recursos como la energía o logísticos; es decir, buscando no solamente reducción de costos sino la generación del valor compartido en las operaciones.

La tercera estrategia contemplada para crear valor compartido, es facilitar el desarrollo de clústeres locales. Estos deben buscar atender las características y demandas reales de la comunidad en las que se desarrollen, pues las condiciones estructurales que éstos presenten, pueden ser determinantes para jugar a favor o en contra de cada empresa. En consecuencia, cada empresa que forma parte del clúster tiene la oportunidad de hacer una invaluable contribución al ayudar a potenciar dichos clústeres locales, a través de sus iniciativas.

\section{ASPECTOS CLAVES QUE FAVORECEN UNA TRANSFORMACIÓN ORGANIZACIONAL HACIA EL VALOR COMPARTIDO}

En los procesos de transición hacia el valor compartido, Porter y Kramer identifican los siguientes aspectos: En primera instancia, es requisito primordial un sólido compromiso de la alta dirección de la empresa y una aproximación integral con el objeto de comprometer a todos los empleados y colaboradores de la organización. 
Sobresalen la interdependencia y las conversaciones generativas, el espíritu emprendedor y la observación permanente del entorno con un marco más amplio, la convivencia con la incertidumbre, la innovación aplicada a gran escala y, sobre todo, el desarrollo del liderazgo, entendiéndolo no como un atributo individual sino como la capacidad de movilizar a un grupo de individuos para gestionar un reto transformador de alto impacto.

La orientación particular hacia las iniciativas en esta línea, comienzan cuando la presidencia de la empresa se apropia del proceso y facilita la conformación de un equipo de dirección con capacidad para tomar decisiones estratégicas y asignar recursos.

\section{Acercamiento a la identificación de las problemá- ticas sociales}

El punto de partida para el valor compartido es identificar y priorizar algunos problemas sociales específicos que representen oportunidades para aumentar los ingresos o reducir los costos. Esto significa un análisis sistemático de las necesidades y brechas sociales no satisfechas y un análisis de cómo estas, se superponen con el negocio a través de los tres niveles de valor compartido. El resultado de este paso es una lista priorizada de las problemáticas sociales que puede abordar una estrategia de valor compartido.

Tras identificar el posible impacto social en uno o más de los tres niveles, que son: reconcebir productos y mercados, redefinición de la productividad en la cadena de valor, y desarrollo de clústeres locales, el paso siguiente es elaborar una justificación sólida del caso basada en una investigación y análisis de cómo el mejoramiento en materia social afectará directamente el desempeño del negocio.

Esta etapa incluye, identificar los objetivos y especificar las actividades y costos involucrados en cada oportunidad de valor compartido, modelar el negocio potencial y los resultados sociales relacionados con los costos (es decir, el potencial de creación de valor) y tomar la decisión de seguir o no con el proyecto.

\section{OBSTÁCULOS Y DESAFÍOS PARA LA IMPLEMENTACIÓN DE LA RESPONSABILIDAD SOCIAL EMPRESARIAL EN LAS MYPES DE PERÚ Y MÉXICO}

Hablar de RSE en el Perú, sin hablar de las MYPES no sería abordar la realidad peruana, pues de acuerdo a los datos del Instituto Nacional de Estadística e Informática, en el 2013 el Perú registraba 1 millón 713 mil empresas, de las cuales el 99.6\% la conformaban las micro, pequeñas y medianas empresas y tan solo el $0.4 \%$ estaba compuesto por transnacionales.

De estas estadísticas, los resultados por segmentos empresariales, reportaron que de este porcentaje total de $99.6 \%$, un porcentaje de $96.2 \%$ concentraba solo a las micro empresas, el $3.2 \%$ a las pequeñas empresas y el $0.2 \%$ a las medianas empresas; según el tipo de actividad, se reportó que el $44.4 \%$ de las micro empresas se dedicaban a la comercialización de bienes mientras que el $16.2 \%$ de microempresas prestaban servicios administrativos, de apoyo y servicios personales. Sin embargo, en lo que corresponde a la participación en las ventas, otra es la lectura ya que aquí, las grandes empresas reflejaban en ventas un 79.3\%; así mismo, en lo que se refiere a índices de empleabilidad, las MYPES generan más del $80 \%$ de la empleabilidad aportando con su actividad, emprendimiento y creatividad en más del $60 \%$ del PBI; es por ello que el papel de las MYPES es considerado decisivo y clave para el desarrollo del país, siendo fuente de una RSE para el Perú.

Sin embargo, pese a estas cifras que le otorgan protagonismo a las MYPES, también la estadística reporta el nacimiento anual de unas 300 mil nuevas MYPES; así como, el cierre de unas 200 mil por la falta de apoyo en la gestión a las ya existentes y consolidadas.

También es una realidad de que muchas de las MYPES no están debidamente formalizadas y esto es un problema actual, razón por la que el Estado intervino con la promulgación de una nueva Ley MYPES 
para la simplificación y formalización de las mismas, desde el Ministerio de la Producción y Fomento del Empleo. Sin embargo, el tema de la formalización no es el único ni el principal.

Al cierre del primer trimestre 2015, los resultados de un sondeo realizado por la Cámara de Comercio de Lima con pequeños empresarios, se destaca que son los sobrecostos laborales (26\%) y la informalidad (26\%) los factores que más desalientan la creación de la pequeña y mediana empresa; así mismo, otras barreras fueron la carga tributaria $(22 \%)$ y las barreras burocráticas (13\%). Tomando en consideración las dificultades indicadas por los emprendedores se requiere por parte delEstado una mayor participación en pro de la solución de estos problemas.

Por lo anteriormente referido en lo que concierne a la Responsabilidad Social Empresarial en el Perú, se presentan los desafíos de llegar primero a la mayor formalización de las MYPES, trabajar en una mayor articulación de las mismas en el tejido empresarial nacional y brindarles las herramientas para la competitividad y la asociatividad para su crecimiento y desarrollo sostenible.

México por su parte, enfrenta diversos obstáculos para el avance de la Responsabilidad Social, la principal es la falta de información y la difusión de la relevancia que adquiere en el sector empresarial la ejecución de acciones de RS.

Para ello, resulta fundamental adoptar una acepción moderna del concepto de Responsabilidad Social, en la cual el cumplimiento de la legalidad, la transparencia, los principios éticos, el diálogo y la concertación con todos los actores del desarrollo económico y social, así como, la responsabilidad generacional frente a las generaciones venideras $y$ frente al cuidado del medio ambiente, resultan pilares de la empresa socialmente responsable.

Por esta razón, al ser las PYMES la mayoría de las empresas del continente y ser las que generan la mayor parte del empleo, deben tener hoy en día la visión de que la Responsabilidad Social es un valor inherente a la empresa.

\section{PROPÓSITO DE LA MEDICIÓN DEL VALOR COMPARTIDO}

La medición en los últimos años ha cobrado enorme importancia; en especial, con la gestión de los intangibles y dentro de ello, en lo referente a la reputación y la marca.

En lo que respecta al valor compartido su cuantificación del impacto es necesario porque las compañías que no entienden la relación directa entre el valor compartido y la productividad y el negocio, pierden opciones de innovación, crecimiento e impacto social, como lo sostiene el recientemente publicado informe global Measuring shared value, coordinado por Porter y Foundation Strategy Group (FSG), una iniciativa a nivel internacional que busca avanzar en el valor compartido.

Un mecanismo podría ser, utilizar los indicadores de proveedores líderes de herramientas para las decisiones de inversión como Foundation and Ethical Investment Research Services (EIRIS) o Morgan Stanley Capital International, (MSCI) los cuales proporcionan un conjunto de indicadores de sostenibilidad relativos a su gestión medioambiental, social y de gobierno (ESG por sus siglas en inglés) para orientar las decisiones de inversión y guiar la divulgación de los impactos socio ambientales, éticos y de gobierno corporativo de los proyectos de inversión y de las empresas.

\section{LA RESPONSABILIDAD SOCIAL EMPRESARIAL (RSE) EN EL PERÚ}

Desde hace unos diez años atrás, en el Perú se ha venido hablando de Responsabilidad Social Empresarial (RSE).

Se puede afirmar que el proceso de incorporación de políticas en RSE no ha sido progresivo en este país, éste ha sido más bien algo forzado por las exigencias de las empresas multinacionales que traían consigo mayores estándares, en el aspecto medio ambiental; así como, en lo referente al impacto en el ser humano y su entorno como comunidad en cada proyecto, sea 
minero, vial, portuario, hidrocarburos, agroindustrial, servicios, entre otros, dado que el crecimiento del Perú ha sido y sigue siendo vertiginoso, ejemplo de ello son los importantes índices de crecimiento en el sector construcción que representa el 5.2\% del PBI del Perú.

Por todo lo referido, se concluye que en el Perú, el desarrollo del concepto y su implementación en las diversas organizaciones, es todavía incipiente, ya que gran parte del empresariado no identifica con claridad el costo-beneficio de la implementación de un sistema de RSE; no obstante, ello no significa que no se apliquen políticas socialmente responsables en el marco de sus misiones, visiones, valores o que ejecuten acciones en esta línea.

\section{LA RESPONSABILIDAD SOCIAL EMPRESARIAL (RSE) EN MÉXICO}

En los últimos años en México, se han logrado avances importantes con la reinserción de la Responsabilidad Social Empresarial (RSE) en el plan de negocios de las empresas.

De acuerdo con la maestra Martha Elizalde Durán, Coordinadora de Consultoría y Comunicación de la Facultad de Responsabilidad Social de la Universidad Anáhuac Norte, uno de los indicadores que refleja el crecimiento de la RSE en la práctica e iniciativa del sector empresarial es el Distintivo Empresa Socialmente Responsable (ESR), otorgado por el CEMEFI.

En la opinión de Salomón Chertorivski, Secretario de Desarrollo Económico del Distrito Federal , en México se tiene una tarea pendiente en el crecimiento y en el fortalecimiento; es importante que a las empresas se les entiendan y se les asuman no sólo como generadoras de renta, sino como entidades responsables de su entorno y de lo demás en lo que están conectadas.
INTERVENCIÓN DE LA UNIVERSIDAD EN LA RESPONSABILIDAD SOCIAL EMPRESARIAL CON VALOR COMPARTIDO

El sector educativo viene a ser el área donde se ofrece mejores expectativas para el avance en la Responsabilidad Social Empresarial con valor compartido, pues sus graduados ejercerán influencia en las empresas, gobierno y sociedad civil.

Por ello, no solo el más inmediato entorno social es afectado por la universidad, sino que a través de la visión de un profesional, sus ideas acerca del papel que juega en la sociedad y el tipo de decisiones que toma en relación a su disciplina, impactan en un entorno mucho más amplio.

Esto derivará en el cambio de actitudes de quienes se formen dentro de ellas y por ende, en las actitudes que asuma el egresado en su rol profesional con una proyección de orden moral y ética de los valores que este interpretó durante su formación.

\section{INTERPRETACIÓNDEALGUNOSASPECTOS ABORDADOS EN TORNO AL VALOR COMPARTIDO}

\section{En torno a la definición de valor compartido}

Se presenta una discusión por parte de los especialistas en el tratamiento del tema de la Responsabilidad Social, por cuanto si bien se reconoce su importancia estratégica, consideran también que la Responsabilidad Social Empresarial es un tema tan amplio que abarca también este concepto del valor compartido, por lo tanto no era innecesario presentarlo como un nuevo concepto.

El equipo de investigación considerando haber revisado en profundidad las definiciones de RS y RSE, RSC, encuentra coincidencia de fondo en este sentido, reconociendo que en la definición rigurosa de la RSE se contiene el concepto de valor compartido, presentándose la responsabilidad social en un 
sentido más “evolucionado", pues ciertamente la RSE necesita ajustes en su definición pero desafortunadamente para muchas empresas todavía la versión anterior aún no se encuentra consolidada, siendo esta RSE todavía una aspiración.

\section{En torno al posicionamiento de la RSE en las empresas de Perú y México}

La realidad tanto en Perú como en México, en relación a las pequeñas y medianas empresas, es que la responsabilidad social empresarial se desarrolla de una forma muy distinta a la de las grandes empresas.

Sin embargo, hay expectativas de que el progreso mejore en este tema, por lo pronto también se viene apreciando que los medios de comunicación están empezando a entender el movimiento y están tratando de apoyarlo en su difusión; así mismo, la universidad también está poniendo de su parte para hacer frente a esta demanda social y de los estudiantes en particular; no obstante se está avanzando poco a poco en varios frentes aunque a ritmos desiguales. En este sentido, se presenta el desafío de profundizar las acciones en torno a impulsar y desarrollar una cultura de responsabilidad social a nivel del sector empresarial, en el sector educativo, haciendo que se tome conciencia de que la responsabilidad social empresarial con valor compartido y la buena gestión son sinónimos.

\section{En torno a la formación de un empresariado consciente y ético}

Las definiciones en torno a la Responsabilidad Social Empresarial refieren el compromiso continuo de las empresas a comportarse éticamente y contribuir al desarrollo económico, mejorando la calidad de vida de los trabajadores y sus familias, así como de la comunidad local y de la sociedad.

Ello, supone la importancia de la integración e involucramiento que asuma la persona o la empresa con el medio ambiente y la sociedad a través de sus metas $y$ acciones considerando su efecto en el entorno.

Esto parece sencillo de cumplir, no obstante dadas las complejidades de los conflictos éticos de los tiempos actuales, es necesario la mejora de la calidad de la formación de los futuros directivos y empresarios tanto de Perú como de México, a efectos de que los futuros empresarios egresen con las herramientas esenciales que les permitan gestionar una responsabilidad social empresarial con valor compartido.

\section{CONCLUSIONES}

1. Crear valor compartido es la forma más evolucionada de aplicar la Responsabilidad Social Empresarial y no se trata de asignar una parte del presupuesto a proyectos de beneficio social, sino, de buscar la manera de hacer negocios de forma que también beneficie a la sociedad.

2. El desarrollo del concepto de valor compartido como su implementación en las organizaciones empresariales de Perú y México, es todavía incipiente pues los empresarios no visualizan claramente el costo-beneficio de la implementación de un Sistema de Responsabilidad Social Empresarial.

3. En el Perú y México, todavía los aspectos de innovación y de responsabilidad social no son determinantes para medir la reputación de una empresa y ello obedece a que aún hace falta un mayor grado de conocimiento y madurez.

4. La medición del valor compartido proporciona una hoja de ruta para alcanzar la promesa del valor compartido, un imperativo global en un momento en el que innovar para alcanzar soluciones sostenibles para los problemas más graves del mundo requiere de lo mejor que los negocios pueden ofrecer.

\section{REFERENCIAS BIBLIOGRÁFICAS}

1. ARIAS, Y. \& LINARES, M. (2008) Desarrollo de un reporte de sostenibilidad basado en la Metodología del Global Reporting Initiative (GRI) aplicado a Ciencias e Ingeniería de la Universidad Católica, Lima.

2. DÍAZ C.\& BUSINESS CÁCERES, N. (2013) El valor compartido como nueva estrategia.

3. LÓPEZ, S. (2011) El razonamiento bioético y la orientación hacia la Responsabilidad Social Empresarial en los estudiantes de negocios. Costa Rica. 
4. MARTÍNEZ N.D.\& AGUIRRE H:F. (2012). Valor compartido como estrategia competitiva en las organizaciones.

5. MAZA G., GARCÍA L.K., SELVAS, H., G.G. GÓMEZ G. (2012) Las micro, pequeñas empresas en Tuxtla Gutiérrez Chiapas de la permanencia y desarrollo. México.

6. MELÉ Doménec Carné. (2007). Responsabilidad social de la empresa: Una revisión crítica a las principales teorías. Ekonomiaz Revista vasca de Economía.

7. Norma Internacional de Normalización ISO 26000 Visión General del Proyecto.

8. PÉREZ y P. I.J. (2009) La Responsabilidad Social en las Empresas Mexicanas. México.
9. PORTER M., \& KRAMER M. (2011). La creación de valor compartido. Harvard Business Review America Latina.

10. PORRAS, N. (2014). Situación de la Responsabilidad Social en México, México D. F.

11. VALLAEYS, F. (2014) La responsabilidad social universitaria: Un nuevo modelo universitario contra la mercantilización. Revista Iberoamericana de Educación Superior (RIES), México, UNAM-IISUE/Universia, vol. V, núm. 12, pp. 105-117, http://ries.universia.net/index.php/ ries/article/view/439. 